\title{
Average intragranular misorientation trends in polycrystalline materials predicted by a viscoplastic self-consistent approach
}

\author{
Ricardo A. Lebensohn (1) ${ }^{\dagger}$, Miroslav Zecevic (1,2), Marko Knezevic (2), Rodney J. McCabe (1) \\ (1) Materials Science and Technology Division, Los Alamos, NM 87544, USA \\ (2) Department of Mechanical Engineering, University of New Hampshire, Durham, NH 03824 \\ USA
}

\begin{abstract}
This work presents estimations of average intragranular fluctuations of lattice rotation rates in polycrystalline materials, obtained by means of the viscoplastic self-consistent (VPSC) model. These fluctuations give a tensorial measure of the trend of misorientation developing inside each single crystal grain representing a polycrystalline aggregate. We first report details of the algorithm implemented in the VPSC code to estimate these fluctuations, which are then validated by comparison with corresponding full-field calculations. Next, we present predictions of average intragranular fluctuations of lattice rotation rates for cubic aggregates, which are rationalized by comparison with experimental evidence on annealing textures of fcc and bcc polycrystals deformed in tension and compression, respectively, as well as with measured intragranular misorientation distributions in a $\mathrm{Cu}$ polycrystal deformed in tension. The orientation-dependent and micromechanically-based estimations of intragranular misorientations that can be derived from the present implementation are necessary to formulate sound submodels for the prediction of quantitatively accurate deformation textures, grain fragmentation, and recrystallization textures using the VPSC approach.
\end{abstract}

Keywords: Polycrystal plasticity modeling, Micromechanics, Misorientation, Texture, Recrystallization.

${ }^{+}$Corresponding author: Ricardo A. Lebensohn, Materials Science and Technology Division, Los Alamos National Laboratory, MS G755, Los Alamos, NM 87845, USA. Phone \#: +1-505-6653035, Fax \#: +1-505-667-8021, e-mail: lebenso@lanl.gov 


\section{1- Introduction}

The 1-site viscoplastic (VP) self-consistent (SC) theory [1,2] is a continuum-level, microstructure-sensitive polycrystal plasticity formulation that has been widely adopted by the Mechanics of Materials community for computing the mechanical behavior, texture and microstructure evolution of polycrystalline materials. The VPSC code [3] is nowadays extensively used to simulate plasticity of polycrystalline aggregates based on the knowledge of deformation mechanisms at the single crystal level and validated by experiments on metals, minerals and polymers. Beyond numerous applications involving single material point calculations for simple geometries and boundary conditions, VPSC has recently been adapted to account for microstructural effects in multiscale calculations either by coupling it directly with Finite Elements (FE) (e.g. [4]) or by using VPSC-based fitting of anisotropic yield functions for subsequent use in the FE analysis (e.g. [5]). These new developments greatly expanded the scope of applications of the model.

VPSC balances efficiency - the number of degrees of freedom of the model, given by the number of mechanical phases, i.e. single crystal orientations or "grains" representing the polycrystal, typically does not exceed a few thousand; representativity - the predicted mechanical response is statistically representative of a large volumetric average over the aggregate; and ability to capture the underlying physical mechanisms of plastic deformationgeometry, activation and hardening of slip and twinning, texture and grain morphology-induced anisotropy, etc. For these reasons, VPSC is usually the best compromise compared with the more efficient but simplistic iso-strain Taylor formulation, and the fully space-resolved but more computational intensive full-field crystal plasticity (CP) formulations like CP-FEM (e.g. [6]) or FFT-based formulations (e.g. VPFFT [7,8]). There are, however, known limitations of the standard VPSC formulation, some of which have been mitigated at the expense of more sophisticated theoretical approaches and numerical implementations.

One of these limitations is related to the necessary approximation involved in applying the SC formulation to non-linear material behavior. The SC theory was originally formulated for linear elastic materials [9]. For non-linear aggregates deforming in the viscoplastic regime, several differing SC approximations are used to linearize the non-linear mechanical behavior at the single crystal level. First-order SC formulations (known as secant [10], tangent [2], and affine 
[11] approximations) are based on linearization schemes that make use of relations between average field quantities only, disregarding higher-order statistical information of the micromechanical fields inside the mechanical phases. This assumption has been proven to be questionable [12-14], especially when strong directionality and large variations in local properties are expected, like in the cases of low rate-sensitivity materials, aggregates made of highly anisotropic grains, and multiphase polycrystals. In these cases, strong deformation gradients are likely to develop inside grains and the use of first moments of the micromechanical field distributions to linearize the behavior at single crystal level is insufficient. To overcome this limitation, a more accurate non-linear homogenization method, Ponte Castañeda's second-order approach [15-16], based on a linearization scheme that incorporates information on the second moments (average fluctuations) of the field distributions in each mechanical phase, has been added to VPSC [14]. A key aspect of this extension has been the implementation of an algorithm to calculate second moments of the stress field distribution inside each representative grain drawing upon [17-19], which is now available in the VPSC code [3].

Another limitation of the standard VPSC formulation is related to the fact that only the average values (first moments) of the micromechanical fields inside the mechanical phases are used to update the microstructure. In particular, the average lattice rotation calculated for each grain is applied to update its average orientation after each deformation increment, and the evolution of deformation texture is based on these average orientation updates. This approximation, which neglects the progressive build-up of intragranular orientation dispersion, has two deleterious effects on the quality of the predicted texture and microstructure evolution. First, the predicted deformation textures are systematically sharper than the experimental textures (e.g. $[7,20])$. This becomes a concern especially when the VPSC model is intended to quantitatively describe texture-induced anisotropy, e.g. in coupled FE-VPSC multiscale calculations. Second, since intragranular misorientation is not accounted for, phenomena like grain size reduction by grain fragmentation, and other important mechanisms affecting microstructure evolution that are driven in part by local lattice misorientation like recovery and recrystallization cannot be predicted based on strictly micromechanical considerations. Different ad-hoc, highly empirical models have been proposed to supplement VPSC calculations to predict smoother textures (e.g. [21]), grain fragmentation (e.g. [22]) and recovery and recrystallization (e.g. [23]). 
In this work we demonstrate how the numerically tractable problem of estimating average fluctuations of the stress field distribution inside each mechanical phase, already implemented in the VPSC code as part of the second-order linearization procedure, can be used to calculate the corresponding second moments of the lattice rotation rate field in each grain.

In this initial contribution, we report the algorithm to calculate these average lattice rotation rate fluctuations in detail, and present results on axisymmetric deformation of cubic polycrystals. These are compared with experimental annealing textures of fcc and bcc polycrystals deformed in tension and compression, respectively, as well as with measured intragranular misorientation distributions in $\mathrm{Cu}$ polycrystals deformed in tension. In a future contribution [24], estimations of intragranular misorientations derived from these average fluctuations in lattice rotation rate will be applied to formulate sound models for the prediction of quantitatively accurate deformation textures, grain fragmentation, and recrystallization textures.

\section{2- Model}

In what follows, the inner products and the outer product between two tensors are denoted by ":" (twice contracted), “::" (four times contracted), and “ $\otimes$ ", respectively. The viscoplastic constitutive behavior at each material point $\mathbf{x}$ deforming by slip is described by means of the following non-linear equation:

$$
\dot{\varepsilon}(\mathbf{x})=\sum_{\mathrm{k}} \mathrm{m}^{\mathrm{k}}(\mathbf{x}) \dot{\gamma}^{\mathrm{k}}(\mathbf{x})=\dot{\gamma}_{\mathrm{o}} \sum_{\mathrm{k}} \mathrm{m}^{\mathrm{k}}(\mathbf{x})\left(\frac{\mathrm{m}^{\mathrm{k}}(\mathbf{x}): \sigma(\mathbf{x})}{\tau_{\mathrm{o}}^{\mathrm{k}}(\mathbf{x})}\right)^{\mathrm{n}} \operatorname{sgn}\left(\mathrm{m}^{\mathrm{k}}(\mathbf{x}): \sigma(\mathbf{x})\right)
$$

where $\tau_{\mathrm{o}}^{\mathrm{k}}$ is the threshold resolved shear stress of slip system (k) (adopted to have a value 1 in arbitrary stress units, in all calculations that follow), $m_{i j}^{k}=\frac{1}{2}\left(n_{i} b_{j}^{k}+n_{j}^{k} b_{i}^{k}\right)$ is the symmetric Schmid tensor associated with slip system $(\mathrm{k})$, where $\mathrm{n}^{\mathrm{k}}$ and $\mathrm{b}^{\mathrm{k}}$ are the normal and Burgers vector direction of such slip system, and $\dot{\varepsilon}$ and $\sigma$ are the deviatoric strain rate and stress. The local shear rate on slip system $(\mathrm{k}), \dot{\gamma}^{\mathrm{k}}$, is given by: 
$\dot{\gamma}^{\mathrm{k}}(\mathbf{x})=\dot{\gamma}_{\mathrm{o}}\left(\frac{\tau^{\mathrm{k}}(\mathbf{x})}{\tau_{\mathrm{o}}^{\mathrm{k}}(\mathbf{x})}\right)^{\mathrm{n}} \operatorname{sgn}\left(\tau^{\mathrm{k}}(\mathbf{x})\right)=\dot{\gamma}_{\mathrm{o}}\left(\frac{\mathrm{m}^{\mathrm{k}}(\mathbf{x}): \sigma(\mathbf{x})}{\tau_{\mathrm{o}}^{\mathrm{k}}(\mathbf{x})}\right)^{\mathrm{n}} \operatorname{sgn}\left(\mathrm{m}^{\mathrm{k}}(\mathbf{x}): \sigma(\mathbf{x})\right)$

where $\tau^{\mathrm{k}}$ is the resolved shear stress on slip system (k), $\dot{\gamma}_{\mathrm{o}}$ is a normalization factor (adopted to be $1 \mathrm{~s}^{-1}$ in all calculations that follow), and the stress exponent $n$ is the inverse of the ratesensitivity. If the shear rates are known, the lattice rotation rate or plastic spin associated with slip activity at single crystal material point $\mathbf{x}$ is given by:

$-\dot{\omega}^{\mathrm{p}}(\mathbf{x})=-\sum_{\mathrm{k}} \alpha^{\mathrm{k}}(\mathbf{x}) \dot{\gamma}^{\mathrm{k}}(\mathbf{x})$

where $\alpha_{i j}^{k}=\frac{1}{2}\left(n_{i}^{k} b_{j}^{k}-n_{j}^{k} b_{i}^{k}\right)$ is the antisymmetric Schmid tensor.

Let us assume linear relations as approximations of the actual non-linear relations, Eqs. (1-2), in the mechanical phase $(r)$ :

$\dot{\varepsilon}(\mathbf{x})=\mathrm{M}^{(\mathrm{r})}: \sigma(\mathbf{x})+\dot{\varepsilon}^{\mathrm{o}(\mathrm{r})}$

$\dot{\gamma}^{\mathrm{k}}(\mathbf{x})=\eta^{\mathrm{k}(\mathrm{r})} \tau^{\mathrm{k}}(\mathbf{x})+\dot{\mathrm{g}}^{\mathrm{ok}(\mathrm{r})}$

where the moduli $\mathrm{M}^{(\mathrm{r})}$ and $\dot{\varepsilon}^{\mathrm{o}(\mathrm{r})}$ are the compliance and back-extrapolated strain rate (strain rate under zero stress) of grain (r), respectively, and $\eta^{\mathrm{k}(\mathrm{r})}$ and $\dot{\mathrm{g}}^{\mathrm{ok}(\mathrm{r})}$ are the slip-level compliance and back-extrapolated shear rate, respectively. Depending on the linearization assumption, the above moduli can be chosen differently. For example, under the affine linearization the moduli are given by:

$$
\begin{aligned}
& \mathrm{M}^{(\mathrm{r})}=\mathrm{n} \dot{\gamma}_{\mathrm{o}} \sum_{\mathrm{k}} \frac{\mathrm{m}^{\mathrm{k}(\mathrm{r})} \otimes \mathrm{m}^{\mathrm{k}(\mathrm{r})}}{\tau_{\mathrm{o}}^{\mathrm{k}(\mathrm{r})}}\left(\frac{\mathrm{m}^{\mathrm{k}(\mathrm{r})}: \sigma^{(\mathrm{r})}}{\tau_{\mathrm{o}}^{\mathrm{k}(\mathrm{r})}}\right)^{\mathrm{n}-1} \\
& \dot{\varepsilon}^{\mathrm{o}(\mathrm{r})}=(1-\mathrm{n}) \dot{\gamma}_{\mathrm{o}} \sum_{\mathrm{k}}\left(\frac{\mathrm{m}^{\mathrm{k}(\mathrm{r})}: \sigma^{(\mathrm{r})}}{\tau_{\mathrm{o}}^{\mathrm{k}(\mathrm{r})}}\right)^{\mathrm{n}} \operatorname{sgn}\left(\mathrm{m}^{\mathrm{k}(\mathrm{r})}: \sigma^{(\mathrm{r})}\right)
\end{aligned}
$$




$$
\begin{aligned}
& \eta^{\mathrm{k}(\mathrm{r})}=\mathrm{n} \frac{\dot{\gamma}_{\mathrm{o}}}{\tau_{\mathrm{o}}^{\mathrm{k}(\mathrm{r})}}\left(\frac{\tau^{\mathrm{k}(\mathrm{r})}}{\tau_{\mathrm{o}}^{\mathrm{k}(\mathrm{r})}}\right)^{\mathrm{n}-1} \\
& \dot{\mathrm{g}}^{\mathrm{ok}(\mathrm{r})}=(1-\mathrm{n}) \dot{\gamma}_{\mathrm{o}}\left(\frac{\tau^{\mathrm{k}(\mathrm{r})}}{\tau_{\mathrm{o}}^{\mathrm{k}(\mathrm{r})}}\right)^{\mathrm{n}} \operatorname{sgn}\left(\tau^{\mathrm{k}(\mathrm{r})}\right)
\end{aligned}
$$

where the supra-index (r) on the magnitudes on the right-hand side of these equations indicates average over the mechanical phase (r), e.g. $\sigma^{(\mathrm{r})}=\langle\sigma(\mathbf{x})\rangle^{(\mathrm{r})}=\langle\sigma\rangle^{(\mathrm{r})}$.

Performing homogenization on a linearized heterogeneous medium whose local behavior is described by Eq. (4) results in an analogous linear relation at the effective medium (macroscopic) level:

$\dot{\mathrm{E}}=\overline{\mathrm{M}}: \Sigma+\dot{\mathrm{E}}^{\mathrm{O}}$

where $\dot{\mathrm{E}}$ and $\Sigma$ are overall (macroscopic) magnitudes and $\overline{\mathrm{M}}$ and $\dot{\mathrm{E}}^{\mathrm{o}}$ are the macroscopic viscoplastic compliance and back-extrapolated strain rate, respectively. The latter moduli are a priori unknown and need to be adjusted, e.g. using the linear SC method, which gives the following fix-point equations (see [14] for a detailed derivation of these equations):

$$
\begin{aligned}
& \bar{M}=\left\langle M^{(r)}: B^{(r)}\right\rangle=\sum_{r} c^{(r)} M^{(r)}: B^{(r)} \\
& \dot{E}^{o}=\left\langle M^{(r)}: b^{(r)}+\dot{\varepsilon}^{o(r)}\right\rangle=\sum_{r} c^{(r)}\left(M^{(r)}: b^{(r)}+\dot{\varepsilon}^{o(r)}\right)
\end{aligned}
$$

where $\langle\cdot\rangle$ indicates average over the aggregate, with $\mathrm{c}^{(\mathrm{r})}$ being the volume fraction associated with grain (r). The localization tensors $\mathrm{B}^{(\mathrm{r})}$ and $\mathrm{b}^{(\mathrm{r})}$ are functions of the local and macroscopic moduli and the interaction tensor given by:

$$
\widetilde{\mathrm{M}}=(\mathrm{I}-\mathrm{S})^{-1}: \mathrm{S}: \overline{\mathrm{M}}
$$

where S is the Eshelby tensor (see [14] for details). The Eshelby tensor, solution of the micromechanical problem of an ellipsoidal inclusion embedded in an infinite medium [25], appears implicitly in the self-consistent expressions, Eqs. (11-12), because it provides the 
connection between the deformation of each mechanical phase and that of the homogenous equivalent medium (HEM) whose mechanical properties need to be adjusted self-consistently. Once the self-consistent problem has converged, an additional lattice rotation rate $\widetilde{\tilde{\omega}}^{(\mathrm{r})}$, associated with a rigid-body rotation of the ellipsoidal inclusion to maintain compatibility with the HEM is given by [14]:

$\widetilde{\omega}^{(r)}=\Pi: \mathrm{S}^{-1}:\left(\dot{\varepsilon}^{(\mathrm{r})}-\dot{\mathrm{E}}\right)$

where $\Pi$ is the antisymmetric Eshelby tensor, which vanishes when the inclusion is spherical. Therefore, in the absence of macroscopic rigid-body rotations, the lattice rotation rate field is given by:

$\dot{\omega}(\mathbf{x})=\widetilde{\tilde{\omega}}^{(\mathrm{r})}-\dot{\omega}^{\mathrm{p}}(\mathbf{x})$

which reduces to $\dot{\omega}(\mathbf{x})=-\widetilde{\tilde{\omega}}^{\mathrm{p}}(\mathbf{x})$ in the case of spherical grains.

The effective stress potential $\overline{\mathrm{U}}_{\mathrm{T}}$ of a linearly viscous polycrystal with back-extrapolated term, described by Eq. (10), may be written in the form [26, 27]:

$\overline{\mathrm{U}}_{\mathrm{T}}=\frac{1}{2} \overline{\mathrm{M}}::(\Sigma \otimes \Sigma)+\dot{\mathrm{E}}^{\mathrm{O}}: \Sigma+\frac{1}{2} \overline{\mathrm{G}}$

where the additional magnitude $\overline{\mathrm{G}}$ is the energy under zero applied stress, given by:

$\overline{\mathrm{G}}=\sum_{\mathrm{r}} \mathrm{c}^{(\mathrm{r})} \dot{\varepsilon}^{\mathrm{o}(\mathrm{r})}: \mathrm{b}^{(\mathrm{r})}$

The average second moment of the stress field over mechanical phase (r) is a fourth-rank tensor given by:

$$
\langle\sigma \otimes \sigma\rangle^{(\mathrm{r})}=\frac{2}{\mathrm{c}^{(\mathrm{r})}} \frac{\partial \overline{\mathrm{U}}_{\mathrm{T}}}{\partial \mathrm{M}^{(\mathrm{r})}}=\frac{1}{\mathrm{c}^{(\mathrm{r})}} \frac{\partial \overline{\mathrm{M}}}{\partial \mathrm{M}^{(\mathrm{r})}}: \because(\Sigma \otimes \Sigma)+\frac{2}{\mathrm{c}^{(\mathrm{r})}} \frac{\partial \dot{\mathrm{E}}^{\mathrm{O}}}{\partial \mathrm{M}^{(\mathrm{r})}}: \Sigma+\frac{1}{\mathrm{c}^{(\mathrm{r})}} \frac{\partial \overline{\mathrm{G}}}{\partial \mathrm{M}^{(\mathrm{r})}}
$$

The numerical algorithm to calculate the above expression based on derivatives of Eqs. (11-13) and (17) is given in [14].

The second moment of the plastic spin field in mechanical phase (r) is given by: 


$$
\left\langle\dot{\omega}^{\mathrm{p}} \otimes \dot{\omega}^{\mathrm{p}}\right\rangle^{(\mathrm{r})}=\left\langle\left(\sum_{\mathrm{k}} \alpha^{\mathrm{k}}(\mathbf{x}) \dot{\gamma}^{\mathrm{k}}(\mathbf{x})\right) \otimes\left(\sum_{\mathrm{k}} \alpha^{\mathrm{k}}(\mathbf{x}) \dot{\gamma}^{\mathrm{k}}(\mathbf{x})\right)\right\rangle^{(\mathrm{r})}
$$

Assuming crystal orientation is uniform inside grain (r):

$$
\left\langle\dot{\omega}^{\mathrm{p}} \otimes \dot{\omega}^{\mathrm{p}}\right\rangle^{(\mathrm{r})}=\sum_{\mathrm{k}, \mathrm{k}^{\prime}}\left(\alpha^{\mathrm{k}(\mathrm{r})} \otimes \alpha^{\mathrm{k}^{\prime}(\mathrm{r})}\right)\left\langle\dot{\gamma}^{\mathrm{k}} \dot{\gamma}^{\mathrm{k}^{\prime}}\right\rangle^{(\mathrm{r})}
$$

Using the linearized expression for the shear rates (Eq. (5)) the above can be expressed in terms of the average second moment of the stress field $\langle\sigma \otimes \sigma\rangle^{(\mathrm{r})}$ (Eq. (18)) as:

$$
\begin{aligned}
& \left\langle\dot{\omega}^{\mathrm{p}} \otimes \dot{\omega}^{\mathrm{p}}\right\rangle^{(\mathrm{r})}=\left\{\sum_{\mathrm{k}, \mathrm{k}^{\prime}}\left(\eta^{\mathrm{k}(\mathrm{r})} \eta^{\mathrm{k}^{\prime}(\mathrm{r})}\right)\left(\alpha^{\mathrm{k}(\mathrm{r})} \otimes \alpha^{\mathrm{k}^{\prime}(\mathrm{r})}\right) \otimes\left(\mathrm{m}^{\mathrm{k}(\mathrm{r})} \otimes \mathrm{m}^{\mathrm{k}^{\prime}(\mathrm{r})}\right)\right\}::\langle\sigma \otimes \sigma\rangle^{(\mathrm{r})} \\
& +\left\{\sum_{\mathrm{k}, \mathrm{k}^{\prime}}\left(\alpha^{\mathrm{k}(\mathrm{r})} \otimes \alpha^{\mathrm{k}^{\prime}(\mathrm{r})}\right) \otimes\left[\left(\eta^{\mathrm{k}(\mathrm{r})} \dot{\mathrm{g}}^{\mathrm{ok}(\mathrm{r})}\right) \mathrm{m}^{\mathrm{k}(\mathrm{r})}+\left(\eta^{\mathrm{k}^{\prime}(\mathrm{r})} \dot{\mathrm{g}}^{\mathrm{ok}(\mathrm{r})}\right) \mathrm{m}^{\mathrm{k}^{\prime}(\mathrm{r})}\right]\right\}:\langle\sigma\rangle^{(\mathrm{r})} \\
& +\sum_{\mathrm{k}, \mathrm{k}^{\prime}}\left(\alpha^{\mathrm{k}(\mathrm{r})} \otimes \alpha^{\mathrm{k}^{\prime}(\mathrm{r})}\right)\left(\dot{\mathrm{g}}^{\mathrm{ok}(\mathrm{r})} \dot{\mathrm{g}}^{\mathrm{ok}^{\prime}(\mathrm{r})}\right)
\end{aligned}
$$

If the grains are spherical, the average second moment of the total lattice rotation rate is simply:

$$
\langle\dot{\omega} \otimes \dot{\omega}\rangle^{(\mathrm{r})}=\left\langle\dot{\omega}^{\mathrm{p}} \otimes \dot{\omega}^{\mathrm{p}}\right\rangle^{(\mathrm{r})}
$$

Otherwise, using Eq. (15) for the general case of elliptical grains results in:

$$
\begin{aligned}
\langle\dot{\omega} \otimes \dot{\omega}\rangle^{(\mathrm{r})}= & \left\langle\left\langle\left(\widetilde{\dot{\omega}}^{(\mathrm{r})}-\dot{\omega}^{\mathrm{p}}(\mathbf{x})\right) \otimes\left(\widetilde{\dot{\omega}}^{(\mathrm{r})}-\dot{\omega}^{\mathrm{p}}(\mathbf{x})\right)\right\rangle^{(\mathrm{r})}=\right. \\
& =\left\langle\dot{\omega}^{\mathrm{p}} \otimes \dot{\omega}^{\mathrm{p}}\right\rangle^{(\mathrm{r})}+\widetilde{\dot{\omega}}^{(\mathrm{r})} \otimes \widetilde{\dot{\omega}}^{(\mathrm{r})}-\widetilde{\dot{\omega}}^{(\mathrm{r})} \otimes\left\langle\dot{\omega}^{\mathrm{p}}\right\rangle^{(\mathrm{r})}-\left\langle\dot{\omega}^{\mathrm{p}}\right\rangle^{(\mathrm{r})} \otimes \widetilde{\dot{\omega}}^{(\mathrm{r})}
\end{aligned}
$$

Once $\langle\dot{\omega} \otimes \dot{\omega}\rangle^{(\mathrm{r})}$ is calculated by means of Eq. (22) or Eq. (23), the covariance of the lattice rotation rate fluctuations is obtained as:

$$
\left\langle\left(\dot{\omega}-\langle\dot{\omega}\rangle^{(\mathrm{r})}\right) \otimes\left(\dot{\omega}-\langle\dot{\omega}\rangle^{(\mathrm{r})}\right)\right\rangle^{(\mathrm{r})}=\langle\dot{\omega} \otimes \dot{\omega}\rangle^{(\mathrm{r})}-\langle\dot{\omega}\rangle^{(\mathrm{r})} \otimes\langle\dot{\omega}\rangle^{(\mathrm{r})}
$$

Representing the antisymmetric rotation (rates) tensors as the corresponding pseudo-vectors, the above covariance can be expressed as a symmetric matrix. Diagonalization of such covariance 
matrix gives an orthonormal basis of eigenvectors $\left(v_{1}, v_{2}, v_{3}\right)$ and three associated eigenvalues: $\dot{\Omega}_{1}^{(\mathrm{r})} \geq \dot{\Omega}_{2}^{(\mathrm{r})} \geq \dot{\Omega}_{3}^{(\mathrm{r})}$. The square root of these eigenvalues:

$\mathrm{SD}_{\mathrm{i}}^{(\mathrm{r})}(\dot{\omega})=\sqrt{\dot{\Omega}_{\mathrm{i}}^{(\mathrm{r})}}(\mathrm{i}=1,2,3)$

are measures of the standard deviation (SD) of the rotation rate distribution along the rotation axes given by the corresponding eigenvectors. A scalar magnitude of the rate at which intragranular orientations are spreading is given by the geometric mean of these SDs:

$\mathrm{SD}^{(\mathrm{r})}(\dot{\omega})=\sqrt[3]{\mathrm{SD}_{1}^{(\mathrm{r})}(\dot{\omega}) \times \mathrm{SD}_{2}^{(\mathrm{r})}(\dot{\omega}) \times \mathrm{SD}_{3}^{(\mathrm{r})}(\dot{\omega})}$

\section{3- Results and Discussion}

In this section we analyze the orientation dependence of the lattice rotation rate distributions given by Eqs. (27-29) for the cases of axisymmetric deformation of fcc and bcc polycrystals. First, as done in [13] for the assessment of mean-field predictions of stress and strain rate distributions, the statistical parameters describing the lattice rotation rate distributions predicted by means of the above algorithm for spherical grains (Eq. (22)) are validated by comparison with corresponding full-field predictions obtained with the VPFFT model for the case of asymmetric tension of an fcc polycrystal with random texture and equiaxed grains. Next, the lattice rotation rate distributions predicted by VPSC in the cases of random fec polycrystals in tension and bec polycrystals in compression are discussed in light of experimental evidence on annealing textures in both classes of materials. The different recrystallization texture components found near $<112>$ and $<114>$ orientations after annealing of fcc polycrystals previously deformed in tension [2830] and of bec polycrystals in compression [31-32], respectively, coincide with the orientations prone to develop the highest intragranular misorientation during deformation, in each case. Finally, the VPSC predictions are assessed for the case of non-random texture and non-spherical grains (exercising the full algorithm for ellipsoidal grains given by Eq. (23)). For this, lattice rotation rate distributions are calculated for an fcc polycrystal after $25 \%$ tensile strain, updating texture and grain shape. These results are qualitatively compared with corresponding distributions measured by electron backscatter diffraction (EBSD) in an OFHC Cu tensile specimen [33]. 
Figure 1a shows VPSC predictions of the per-grain rotation rate averages and standard deviations normalized by $\overline{\dot{\omega}}=\left\langle\dot{\omega}^{(\mathrm{r})}\right\rangle$, corresponding to an fcc polycrystal made of 400 randomlyoriented spherical grains deforming by $\{111\}<110>$ slip and stress exponent $n=10$, deformed axisymmetrically in tension at an applied strain rate of $1 \mathrm{~s}^{-1}$. Figure $1 \mathrm{~b}$ compares the same statistical parameters obtained by sampling and ensemble averaging full-field predictions of the rotation rate fields, calculated for 50 different periodic Voronoi unit cell realizations, made of the same 400 orientations randomly assigned to the Voronoi grains, with identical constitutive behavior and applied boundary conditions. These fields were computed with the VPFFT approach, a spectral formulation that gives the "exact" solution (within the limitations imposed by the necessary discretization of the problem) of the governing equations of strain compatibility and stress equilibrium (for details on the VPFFT model, including the ensemble averaging procedure and unit cell construction by Voronoi tessellation, see [13] and [8] respectively). Table 1 summarizes the statistical information shown in the figure, reporting the overall minimum, mean and maximum values of the per-grain average and geometric mean SD (Eq. (26)) predicted by both approaches. A more detailed comparison between the shapes of the distributions predicted by both models is given in Table 2, showing the min, mean and max values of the three individual SDs (Eq. (25)) of the per-grain rotation rate distributions.

The comparison between the VPSC predictions and the corresponding full-field statistics shows reasonable agreement. Both models predict very similar orientation dependence of the average rotation rates, and, most importantly, of the average dispersions, with maximum near $<112>$. The overall statistics reported in Table 1 for the per-grain average rotations and geometric mean SDs are also in good agreement, as well as the more detailed information on the shapes of the distributions given in Table 2. The minor differences between VPSC and VPFFT predictions can be ascribed to two reasons. 1) The unavoidable adoption of a linearization scheme in VPSC, affine in this case. As discussed above, this implies an approximation of the actual non-linear response of the material, which the VPFFT solutions strictly account for. In the present case of relatively mild single crystal anisotropy and non-linearity, the results of the first-order affine linearization match reasonable well the reference full-field solutions. Materials with higher local mechanical contrast would require the use of second-order linearization, for more accurate 
results. 2) The imperfect statistical representation of the distributions obtained by ensemble averaging of VPFFT solutions.

With the VPSC predictions duly validated by comparison with full-field reference solutions, let us further illustrate the type of information that can be obtained from computing the orientationdependent lattice rotation rate statistics. Figure 2 shows a stereographic projection using a 2-dash symbol for each of the 400 grains considered in the above-described VPSC calculation for a random fcc polycrystal in tension. The first dash links the initial orientation of each grain (r) with the final average orientation obtained by considering a rotation $\omega=\langle\dot{\omega}\rangle^{(\mathrm{r})} \Delta \mathrm{t}$, where $\Delta \mathrm{t}=0.02$, i.e. corresponding to an overall strain increment of $2 \%$. The second dash, centered in the updated average orientation, links these two orientations: $\omega=\left(\langle\dot{\omega}\rangle^{(\mathrm{r})} \pm \mathrm{SD}_{1}^{(\mathrm{r})}(\dot{\omega}) v_{1}\right) \Delta \mathrm{t}$, reflecting the dispersion associated with the largest SD of the rotation rate distribution. Interestingly, the model predicts that the grains rotating towards the stable orientations from orientations near $<110>$ and from intermediate orientations along the $<001>-<111>$ line have their maximum dispersion along the main rotation direction, i.e. with parts of the grain rotating faster and towards the stable orientation and others lagging behind. For all other orientations, the largest dispersion occurs approximately normal to the main rotation direction.

Axisymmetric tension of fcc polycrystals and compression of bcc polycrystals are material processing routes that develop the same kind of $<100>$ and $<111>$ bimodal texture. Octahedral $\{111\}<110>$ slip is the prevalent deformation mode in fcc polycrystals. If $\{110\}<111>$ slip is considered as the only active slip mode in bcc aggregates, symmetry determines identical local lattice rotations and, consequently, same deformation textures are predicted in the cases of fcc in tension and bcc in compression. However, if pencil glide is considered in bcc, i.e. allowing the possibility of activation of other slip modes with $<111>$ Burgers vectors, namely: $\{112\}<111>$ and $\{123\}<111>$, different micromechanical states are expected. These differences are in general not large enough to change the trend towards the formation of the bimodal $<100>\mid<111>$ deformation textures, but as we will show, they provide a plausible explanation of the different annealing texture components, i.e. $<112>$ orientation appearing in fcc after tensile deformation [28-30], and $<114>$ in bcc after compressive deformation [31-32]. Figure 3 shows the orientation dependence of the average intragranular misorientation trend represented by the per-grain 
geometric mean SD (Eq. (25)) predicted by VPSC for a random fcc polycrystal with 400 spherical grains, deformed in tension by $\{111\}<110>$ octahedral slip, and a random bcc polycrystal with spherical grains with the same orientations, deformed in compression by $\{110\},\{112\},\{123\}<111>$ pencil glide. Note that the color scales are the same in both figures, and that the bcc polycrystal shows smaller overall rotation rate dispersion than the fcc aggregate, consistent with the lower single crystal plastic anisotropy resulting from pencil glide in bcc, compared with octahedral slip in fcc. The regions of orientations prone to develop the maximum average intragranular misorientation, predicted near $<112>$ and $<114>$ for fcc and bcc, respectively, are consistent with the annealing texture components observed experimentally in polycrystalline fcc copper after being deformed in tension [28-30] and in polycrystalline iron after compressive deformation [31-32]. The reason for this is related to the role of the so-called transition bands [34], i.e. regions of high lattice distortion resulting from inhomogeneous deformation, where grains break up into mutually highly misoriented subgrains. During annealing of heavily deformed polycrystalline metals, nucleation of recrystallization is frequently observed to occur in these bands [34].

The trend for the orientation dependence of the average intragranular misorientation shown above in the case of random texture and spherical grains remains qualitatively the same as texture and grain shape evolve. Figure 4 shows the VPSC predictions of the per-grain geometric mean SD after $25 \%$ strain applied to the initially random fec and bcc polycrystals in tension and compression, respectively. In these simulations, texture and grain morphology were updated incrementally according to the average rotations and deformation gradients computed for each grain, resulting in a non-uniform texture and non-spherical grains, after $25 \%$ strain. The consideration of grain morphology evolution in these cases allowed us to exercise the full algorithm to calculate average fluctuations of lattice rotation rates given by Eq. (23). While the bimodal $<001>$ and $<111>$ textures are building up, the grains with the highest average lattice rotation rates remain close to the $<112>$ and $<114>$ orientations in the fcc and bcc cases, respectively. This trend remains the same for simulations carried out up to higher strains, with fewer grains remaining in those orientations exhibiting the maximum average lattice rotation rates.

Another illustrative (albeit still qualitative) comparison of the above simulations for the fcc polycrystal in tension can be carried out with experimental evidence reported by Krog-Pedersen 
et al. [33]. These authors used the EBSD technique on a polished section of a polycrystalline OFHC Cu specimen deformed $25 \%$ in tension to measure the orientation field, which they then processed to obtain the second-rank tensors of second-order central moment of the misorientation distribution for each individual grain identified by EBSD, adopting a 7 deg misorientation criterion to distinguish between grains. Next, they characterized the shape of the misorientation distribution associated with each grain by diagonalization of the above tensors. While these experimentally determined second-order central moment of the misorientation distributions, and the second moment of the lattice rotation rate distributions predicted with VPSC correspond to different fields (total accumulated misorientations vs lattice rotation rates), we expect some correlation between the orientation dependence of the shapes of both distributions, characterized by their respective standard deviations. Krog-Pedersen et al. reported the measured orientation dependence of the misorientation distribution by subdividing the stereographic triangle into four orientation regions (orientations within $10 \mathrm{deg}$ from $<001>, 20$ deg from $<011>$, $15 \mathrm{deg}$ from $<111>$ and the "remaining" orientations), and the shape of the distribution in four cases according to the relative values of the 3 standard deviations, given in Table 3. The most prominent experimental results from [33] are: a) distributions with the largest SD significantly larger than the other two (i.e. "ellipsoid" and "prolate") are predominant for orientations within $10 \mathrm{deg}$ from $<001>$ and $20 \mathrm{deg}$ from $<011>$, and b) "sphere" occur mostly within $15 \mathrm{deg}$ from $<111>$. Figure 5 shows the VPSC predictions of the shape of the distributions of lattice rotation rates according to the ratios between the three SDs and the distribution shape classification of Table 3 for: a) the initial texture, and b) after 25\% strain, accounting for texture and grain morphology evolution. Clearly, the "ellipsoid" distribution of lattice rotation rates is prevalent near $<001>,<011>$ and orientations in between. The accumulation of these lattice rotation rate dispersions integrated through time is consistent with the development of a high proportion of "ellipsoid" and "prolate" misorientation distributions for these orientations, as measured and reported in [33]. Also, while the $<111>$ orientation is dominated by the "oblate" distribution, the "sphere" distribution does occur near $<111>$ although not directly at that orientation. These two distributions of lattice rotation rates integrated and accumulated through time may determine higher probability of finding less anisotropic misorientation distributions, consistent again with Krog-Pedersen et al's measurements near $<111>$. 
The orientation-dependent and micromechanically-based estimations of intragranular misorientations derived from the present implementation may be used to formulate sound submodels for the prediction of quantitatively accurate deformation textures, grain fragmentation, and recrystallization textures using the VPSC approach. For example, one can keep track of orientations representing accumulative deviations from the evolving average grain orientations, e.g. those used to plot the second dashes in Fig. 2, given by the average orientation plus/minus rotations determined by the SDs of the distribution with respect to axes along the corresponding eigenvector directions. Then, a measure of the misorientation accumulated in each grain will be given by the misorientation between these tracked "outlier" orientations and the average orientation. These misorientations can in turn be used to split the original grain into smaller grains with different orientations, capturing grain fragmentation and grain size reduction, as well as effectively smoothing the predicted deformation textures based on micromechanical considerations. Choosing an appropriate threshold misorientation, the grain split would be numerically manageable, occurring after a number of deformation steps instead of after every increment, avoiding a prohibitive geometric increase of the type $2^{\mathrm{k}}$ where $\mathrm{k}$ is the number of deformation steps. Moreover, these average misorientations may be used as mechanical driving forces to predict recrystallization, either dynamically during deformation, or statically after deformation [24].

\section{4- Conclusions}

We have presented the numerical implementation in VPSC of the algorithm to calculate average intragranular fluctuations of lattice rotation rates in polycrystalline materials, which provide a tensorial measure of the trend of misorientation developing inside the grains representing the aggregate. The mean-field VPSC estimations were validated by comparison with corresponding full-field predictions using the VPFFT approach. The agreement between mean-field estimates and ensemble averages of full-field solutions was found to be satisfactory, with minor differences ascribed to the use of a linearization scheme in VPSC, affine in this case, and insufficient statistical information obtained from VPFFT simulations. Average intragranular fluctuations of lattice rotation rates for cubic aggregates were presented and discussed in light of experimental annealing textures of uniaxially deformed fcc and bcc polycrystals. Invoking the concept of recrystallization controlled by nucleation in transition bands, the $<112>$ and $<114>$ 
recrystallization texture components in fcc and bcc metals deformed in tension and compression, respectively, were found to strongly correlate with the orientations tending to develop the largest intragranular misorientation, as predicted by VPSC in each case. Also, the accumulative effect of the predicted shapes of the lattice rotation rate distributions for fcc polycrystals deformed in tension was found to be consistent with measured intragranular misorientation distributions in $\mathrm{Cu}$ polycrystals. The predicted lattice rotation rate distributions can be used to estimate the evolution of average intragranular misorientations, which in turn may contribute to improve texture and microstructure predictions and extend the VPSC approach to model grain fragmentation and recrystallization based on micromechanical considerations.

\section{Acknowledgments}

This work was supported by US Department of Energy, Office of Basic Energy Sciences (OBES) FWP-06SCPE401 and LANL's Laboratory Directed Research and Development (LDRD) Project 20140630ER.

\section{References}

[1] A. Molinari, G.R. Canova, S. Ahzi, Self-consistent approach of the large deformation polycrystal viscoplasticity, Acta Metall. 35 (1987) 2983-2994.

[2] R.A. Lebensohn, C.N. Tomé, A selfconsistent approach for the simulation of plastic deformation and texture development of polycrystals: application to Zirconium alloys, Acta Metall. Mater. 41 (1993) 2611-2624.

[3] C.N. Tomé, R.A. Lebensohn, Manual for Code Viscoplastic Self-Consistent (VPSC), version 7c (http://public.lanl.gov/lebenso/VPSC7c_manual.pdf).

[4] J. Segurado, R.A. Lebensohn, J. LLorca, C.N. Tomé, Multiscale modeling of plasticity based on embedding the viscoplastic self-consistent formulation in implicit finite elements, Int. J. Plast. 28 (2012) 124-140.

[5] B. Plunkett, R. A. Lebensohn, O. Cazacu, F. Barlat, Evolving yield function of hexagonal materials taking into account texture development and anisotropic hardening, Acta Mater. 54 (2006) 4159. 
[6] F. Roters, P. Eisenlohr, L. Hantcherli, D.D. Tjahjanto, T.R. Bieler, D. Raabe, Overview of constitutive laws, kinematics, homogenization and multiscale methods in crystal plasticity finiteelement modeling: Theory, experiments, applications, Acta Mater. 58 (2010) 1152-1211. [7] R.A. Lebensohn, N-site modelling of a 3-D viscoplastic polycrystal using Fast Fourier Transform, Acta Mater. 49 (2001) 2723-2737.

[8] R.A. Lebensohn R. Brenner, O. Castelnau, A.D. Rollett, Orientation image-based micromechanical modelling of subgrain texture evolution in polycrystalline copper, Acta Mater. 56 (2008) 3914-3926.

[9] A.V. Hershey, The elasticity of an isotropic aggregate of anisotropic cubic crystals, J. Appl. Mech. 21 (1954) 236-240.

[10] R. Hill, Continuum micro-mechanics of elastoplastic polycrystals, J. Mech. Phys. Solids 13 (1965) 89-101.

[11] R. Masson, M. Bornert, P. Suquet, A. Zaoui, Affine formulation for the prediction of the effective properties of nonlinear composites and polycrystals, J. Mech. Phys. Solids 48 (2000) 1203-1227.

[12] P. Gilormini, A critical evaluation for various nonlinear extensions of the self-consistent model, in: A. Pineau and A. Zaoui (Eds.), IUTAM Symposium on Micromechanics of Plasticity and Damage of Multiphase Materials, Kluwer Academic Publishers, The Netherlands, 1996, pp. 67-74.

[13] R.A. Lebensohn, Y. Liu, P. Ponte Castañeda, On the accuracy of the self-consistent approximation for polycrystals: comparison with full-field numerical simulations, Acta Mater. 52 (2004) 5347-5361.

[14] R.A. Lebensohn, C.N. Tomé, P. Ponte Castañeda, Self-consistent modeling of the mechanical behavior of viscoplastic polycrystals incorporating intragranular field fluctuations, Phil. Mag. 87 (2007) 4287-4322.

[15] P. Ponte Castañeda, Second-order homogenization estimates for nonlinear composites incorporating field fluctuations. I. Theory, J. Mech. Phys. Solids 50 (2002) 737-757.

[16] Y. Liu, P. Ponte Castañeda, Second-order theory for the effective behavior and field fluctuations in viscoplastic polycrystals, J. Mech. Phys. Solids 52 (2004) 467.

[17] M. Bobeth, G. Diener, Static elastic and thermoelastic field fluctuations in multiphase composites, J. Mech. Phys. Solids 35 (1987) 137-149. 
[18] W. Kreher, Residual stresses and stored elastic energy of composites and polycrystals, J. Mech. Phys. Solids 38 (1990) 115-128.

[19] V.Z. Parton, V.A. Buryachenko, Stress fluctuations in elastic composites, Sov. Phys. Dokl. 35(2) (1990) 191-193.

[20] M. Knezevic, T. Nizolek, M. Ardeljan, I.J. Beyerlein, N.A. Mara, T.M. Pollock, Texture evolution in two-phase $\mathrm{Zr} / \mathrm{Nb}$ lamellar composites during accumulative roll bonding, Int. J. Plast. 57 (2014) 16-28.

[21] C.N. Tomé, R.A. Lebensohn, C.T. Necker, Orientation correlations and anisotropy of recrystallized aluminum, Metall. Mater. Trans. 33A (2002) 2635-2648.

[22] I.J. Beyerlein, R.A. Lebensohn, C.N. Tomé, Modeling of texture and microstructural evolution in the equal channel angular process, Mat. Sci. Eng. A 345 (2003) 122-138. [23] H.R. Wenk, G.R. Canova, Y. Brechet, L. Flandin, A deformation-based model for recrystallization of anisotropic materials, Acta Mater. 45 (1997) 3283-3296.

[24] M. Zecevic, R.J. McCabe, M. Knezevic, R.A. Lebensohn, in preparation.

[25] T. Mura, Micromechanics of Defects in Solids, Martinus-Nijhoff Publishers, Dordrecht, 1987.

[26] N. Laws, On the thermostatics of composite materials, J. Mech. Phys. Solids 21 (1973) 9176.

[27] J.R. Willis, Variational and related methods for the overall properties of composites, Adv. Appl. Mech. 21 (1981) 1-78.

[28] I.L. Dillamore, W.T. Roberts, Preferred orientation in wrought and annealed metals, Metallurgical Reviews 10 (1965) 271-379.

[29] F.J. Humphreys, M. Hatherly, Recrystallization and Related Annealing Phenomena, 2nd ed., Elsevier Ltd., Oxford, 2004, p. 286.

[30] H. Park, D.N. Lee, The evolution of annealing textures in 90 pct drawn copper wire, Metall. Mater. Trans. A 34A (2003) 531-541.

[31] I.L. Dillamore, H. Katoh, The mechanisms of recrystallization in cubic metals with particular reference to their orientation-dependence. Metal. Sci. J. 8 (1974) 73-83.

[32] I.L. Dillamore, H. Katoh, K. Haslam, The nucleation of recrystallisation and the development of textures in heavily compressed iron-carbon alloys, Texture 1 (1974) 151-156. 
[33] S. Krog-Pedersen, J.R. Bowen, W. Pantleon, Quantitative characterization of the orientation spread within individual grains in copper after tensile deformation Int. J. Mat. Res. 100 (2009) 433-438.

[34] I.L. Dillamore, P.L. Morris, C.J.E. Smith, W.B. Hutchinson. Transition bands and recrystallization in metals, Proc. R. Soc. Lond. A 329 (1972) 405-420.

\section{Figure Captions}

Figure 1: VPSC (left) and VPFFT (right) predictions of per-grain (a-b) rotation rate averages $\langle\dot{\omega}\rangle^{(\mathrm{r})}$, and (c-d) geometric mean standard deviations $\mathrm{SD}^{(\mathrm{r})}(\dot{\omega}) / \overline{\dot{\omega}}$, for the case of tensile deformation of an fcc polycrystal made of 400 randomly-oriented spherical/equiaxed grains deforming by $\{111\}<110>$ slip.

Figure 2: Stereographic projection using 2-dash symbols, one for each of the 400 spherical grains representing a random fcc polycrystal deforming by $\{111\}<110>$ slip, as predicted by the VPSC model. First dash links each initial grain orientation with the final average orientation after $2 \%$ tensile strain. Second dash links final average orientation plus/minus a rotation equal to the largest SD of the distribution and rotation axis along the corresponding eigenvector direction. Colors of the symbols correspond to value of the normalized largest SD: $\mathrm{SD}_{1}^{(\mathrm{r})}(\dot{\omega}) / \overline{\dot{\omega}}$.

Figure 3: Orientation dependence of the per-grain geometric mean SD, SD ${ }^{(\mathrm{r})}(\dot{\omega}) / \overline{\dot{\omega}}$ (see Eq. (26)), of lattice rotation rate distributions predicted by VPSC for: (a) a random fec polycrystal with spherical grains, deforming in tension by $\{111\}<110>$ octahedral slip (a random bcc polycrystal with spherical grains deformed in compression by only $\{110\}<111>$ slip results in identical local lattice rotations). (b) A random bcc polycrystal with spherical grains, deforming in compression by $\{110\},\{112\},\{123\}<111>$ pencil glide. Orientations prone to develop maximum average intragranular misorientation are predicted near $<112>$ and $<114>$ for fcc and bcc, respectively.

Figure 4: VPSC predictions of the per-grain geometric mean SD of lattice rotation rate distributions, $\mathrm{SD}^{(\mathrm{r})}(\dot{\omega}) / \overline{\dot{\omega}}$ (see Eq. (26)), after 25\% tensile strain, considering texture and grain morphology evolution (average fluctuations of lattice rotation rates calculated using the full expression for non-spherical grains, Eq. (23)). Cases of: a) fcc polycrystal deforming in tension 
by $\{111\}<110>$ octahedral slip, and b) bcc polycrystal with spherical grains, deforming in compression by $\{110\},\{112\},\{123\}<111>$ pencil glide.

Figure 5: VPSC predictions of the shape of the distributions of lattice rotation rates, according to the ratios between the three $\mathrm{SD}_{\dot{i}}^{(\mathrm{r})}(\dot{\omega})$ (see Table 3), for an fcc polycrystal deforming in tension. Cases of: (a) initial texture, with random orientations and spherical grains, (b) after $25 \%$ strain accounting for texture and grain morphology evolution.

\section{Table Captions}

Table 1: Minimum, mean and maximum values of the per-grain average and geometric mean SD (Eq. (26)) predicted by VPSC and VPFFT

Table 2: Minimum, mean and maximum values of the three individual SDs (Eq. (25)) of the pergrain rotation rate distributions, predicted by VPSC and VPFFT.

Table 3: Classification of the shape of distributions, as a function of the relative values of the 3 standard deviations, according to Krog-Pedersen et al. [33]. 


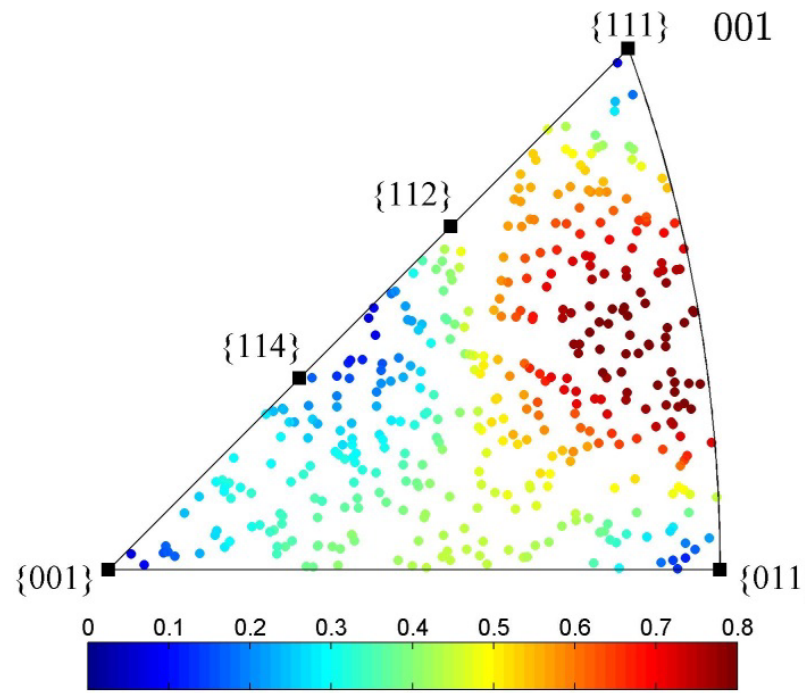

(a) rotation rate averages - VPSC

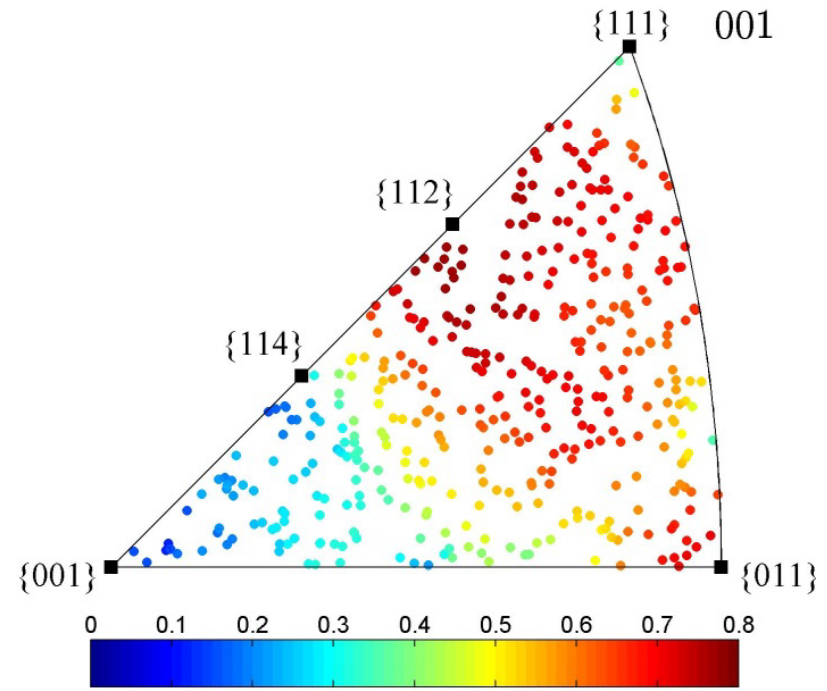

(c) rotation rate SDs - VPSC

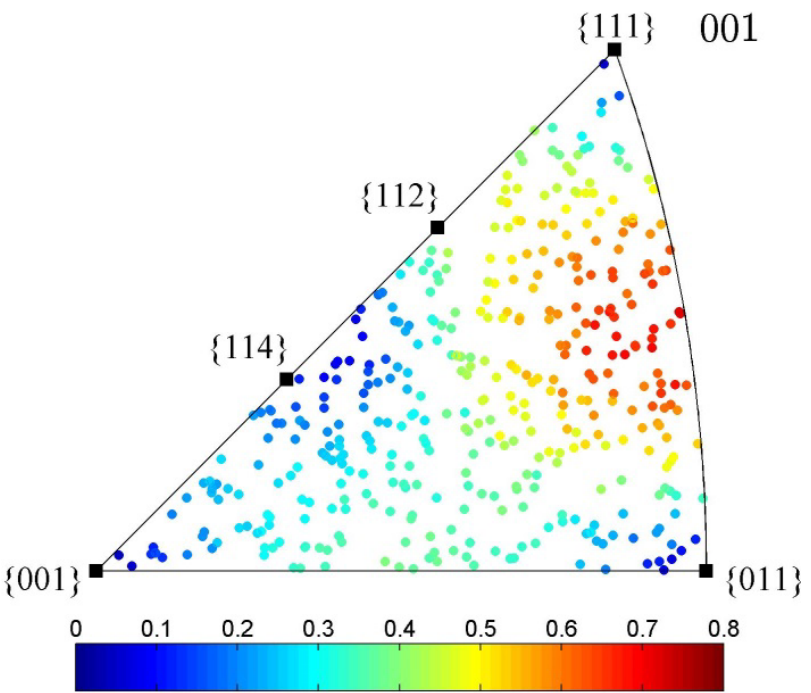

(b) rotation rate averages - VPFFT

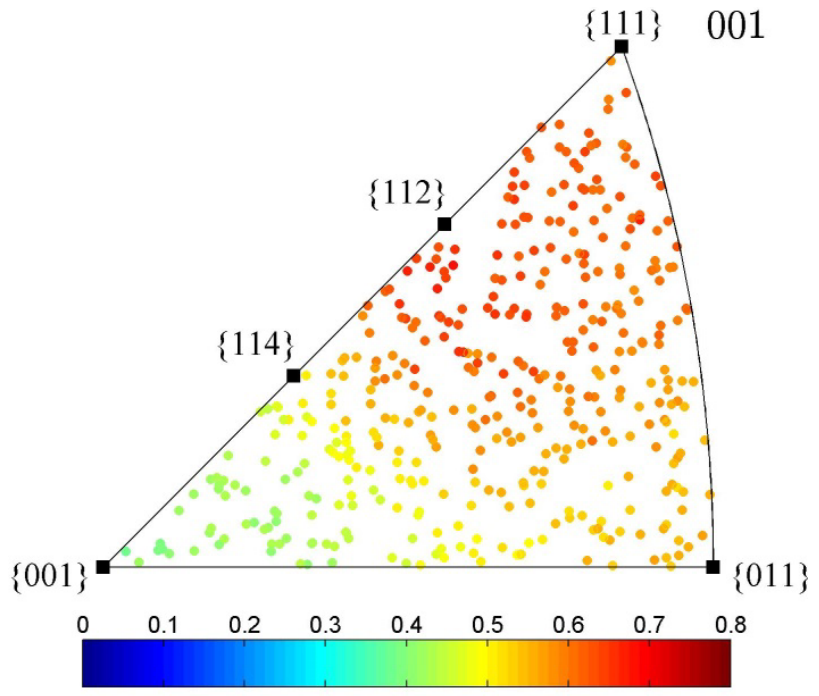

(d) rotation rate SDs - VPFFT

Figure 1: VPSC (left) and VPFFT (right) predictions of per-grain (a-b) rotation rate averages, and (c-d) geometric mean standard deviations, for the case of tensile deformation of an fcc polycrystal made of 400 randomly-oriented spherical/equiaxed grains deforming by $\{111\}<110>$ slip. 


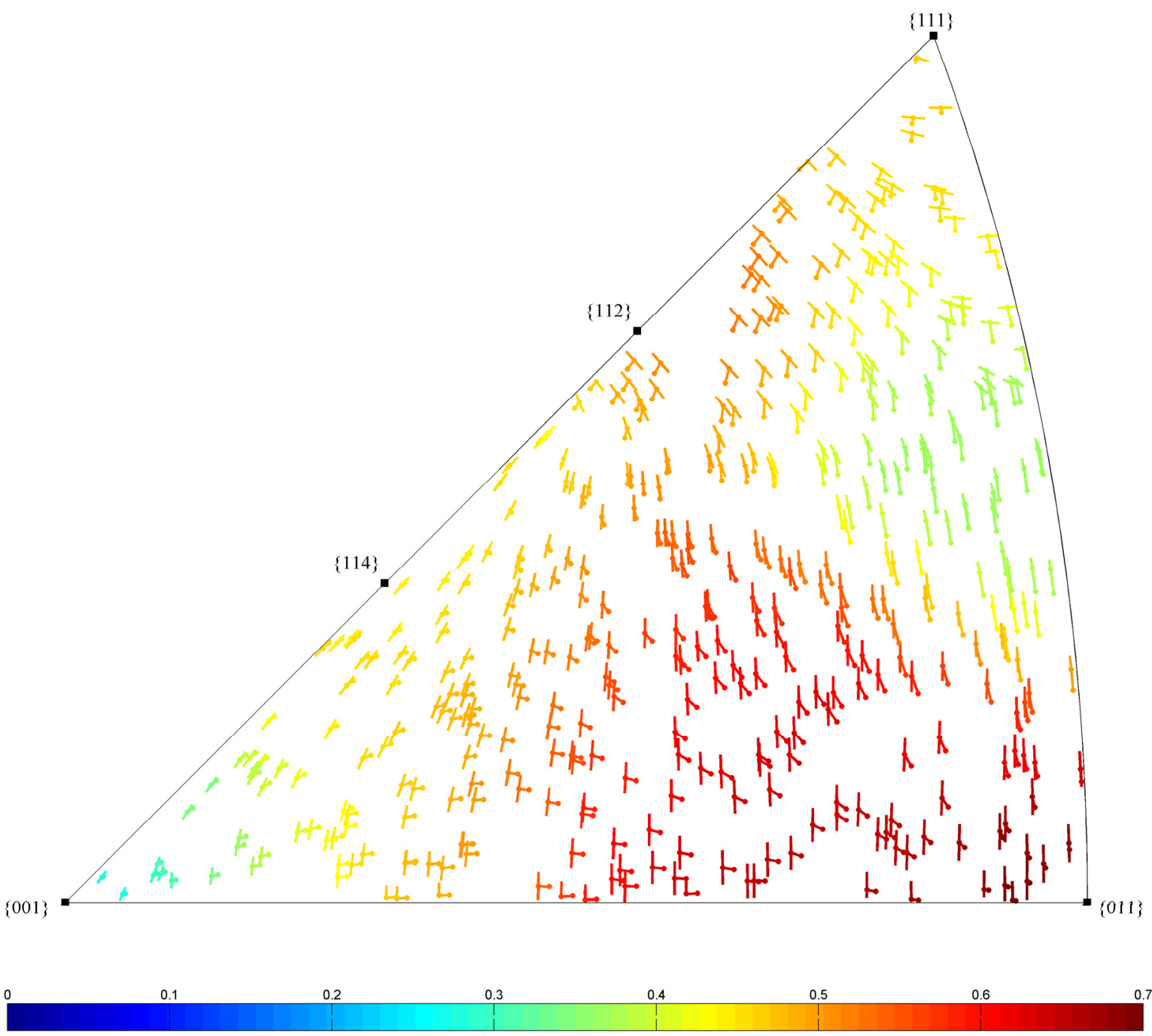

Figure 2: Stereographic projection using 2-dash symbols, one for each of the 400 spherical grains representing a random fcc polycrystal deforming by $\{111\}<110>$ slip, as predicted by the VPSC model. First dash links each initial grain orientation with the final average orientation after $2 \%$ tensile strain. Second dash links final average orientation plus/minus a rotation equal to the largest $\mathrm{SD}$ of the distribution and rotation axis along the corresponding eigenvector direction.

Colors of the symbols correspond to value of the normalized largest SD: $\operatorname{SD}_{1}^{(\mathrm{r})}(\dot{\omega}) / \overline{\dot{\omega}}$. 


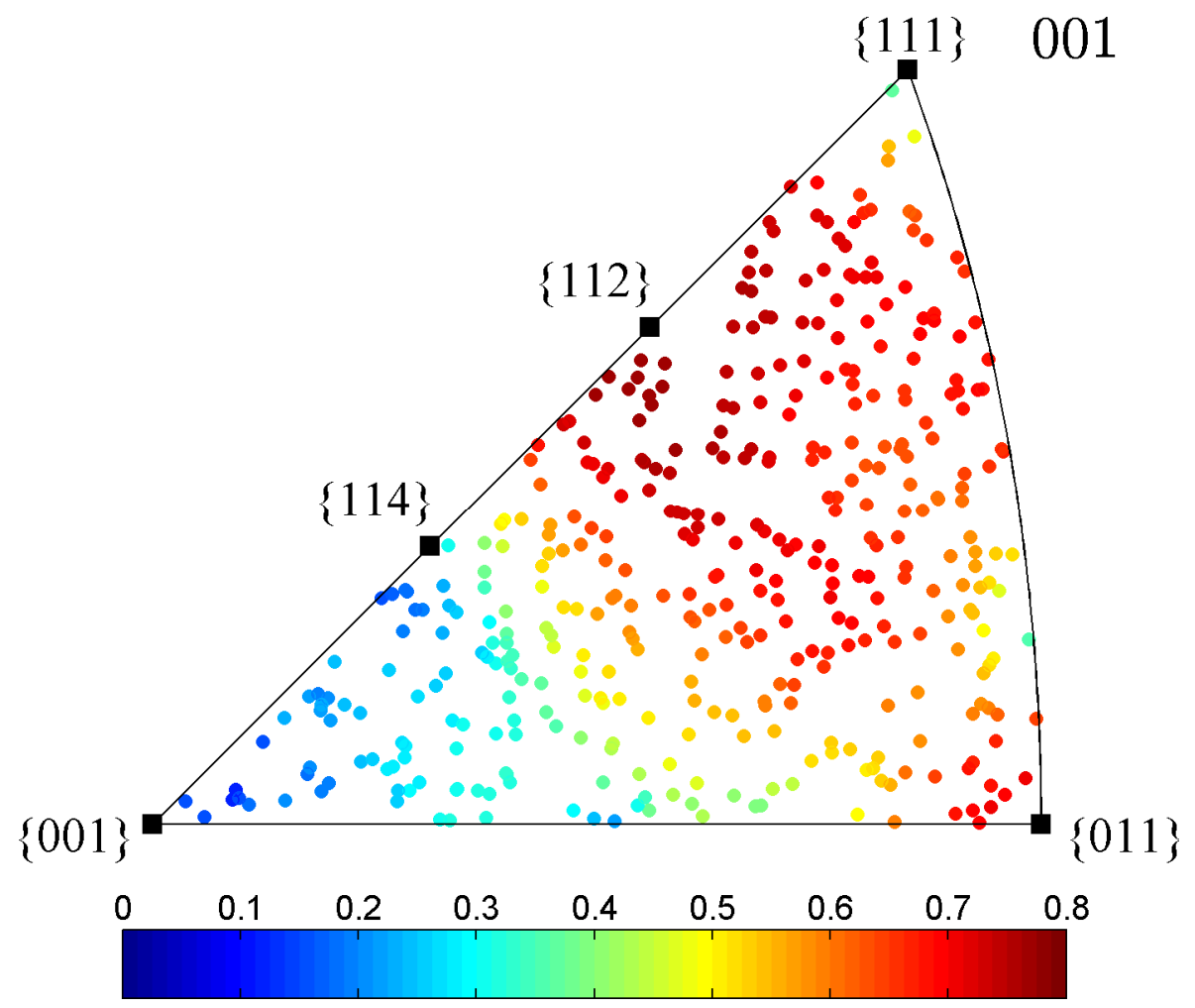

(a) rotation rate SDs - FCC

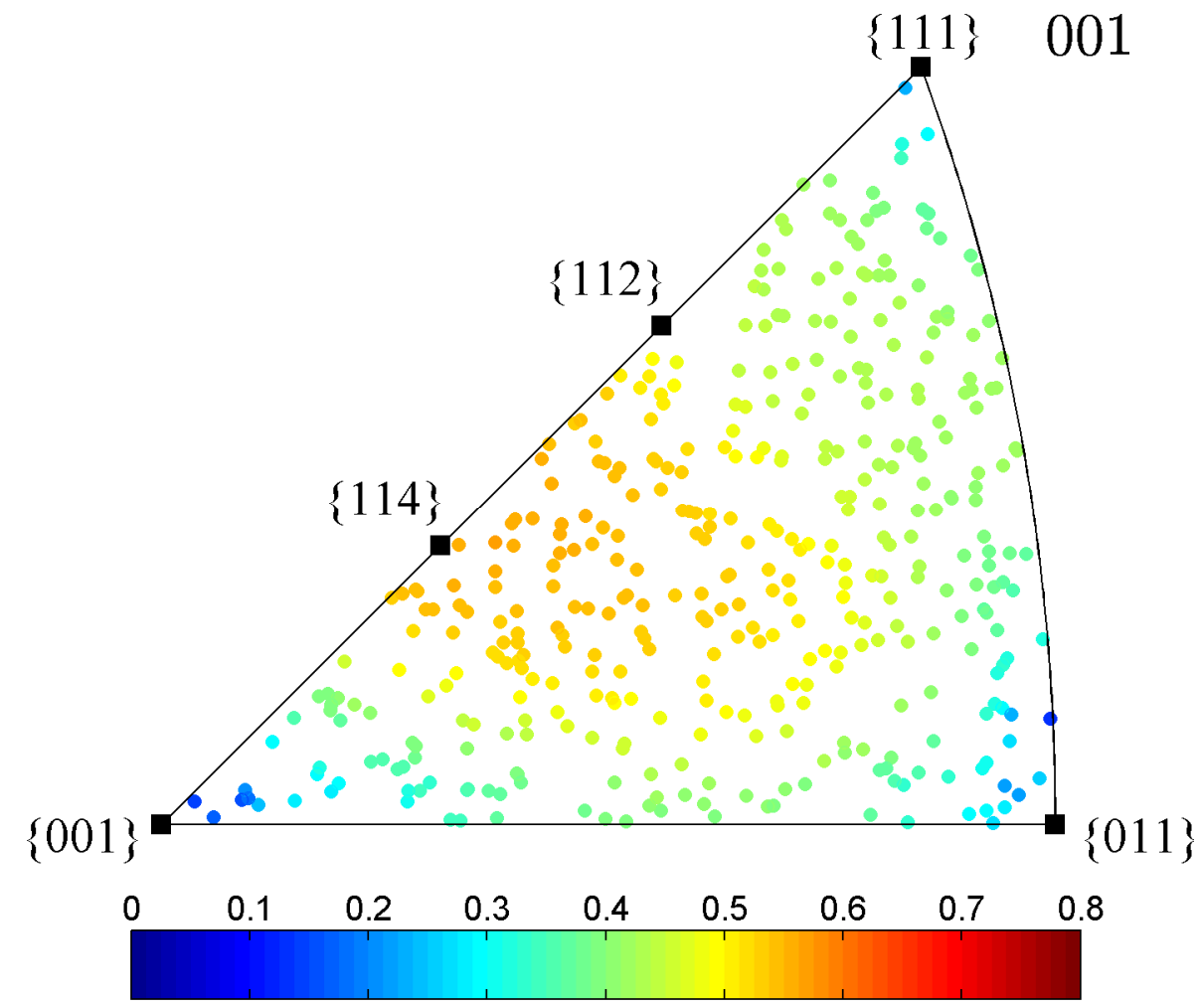

(b) rotation rate SDs - BCC

Figure 3: Orientation dependence of the per-grain geometric mean $\mathrm{SD}, \mathrm{SD}^{(\mathrm{r})}(\dot{\omega}) / \overline{\dot{\omega}}$ (see Eq. (26)), of lattice rotation rate distributions predicted by VPSC for: (a) a random fcc polycrystal with spherical grains, deforming in tension by $\{111\}<110>$ octahedral slip (a random bcc polycrystal with spherical grains deformed in compression by only $\{110\}<111>$ slip results in identical local lattice rotations). (b) A random bcc polycrystal with spherical grains, deforming in compression by $\{110\},\{112\},\{123\}<111>$ pencil glide. Orientations prone to develop maximum average intragranular misorientation are predicted near $<112>$ and $<114>$ for fcc and bcc, respectively. 


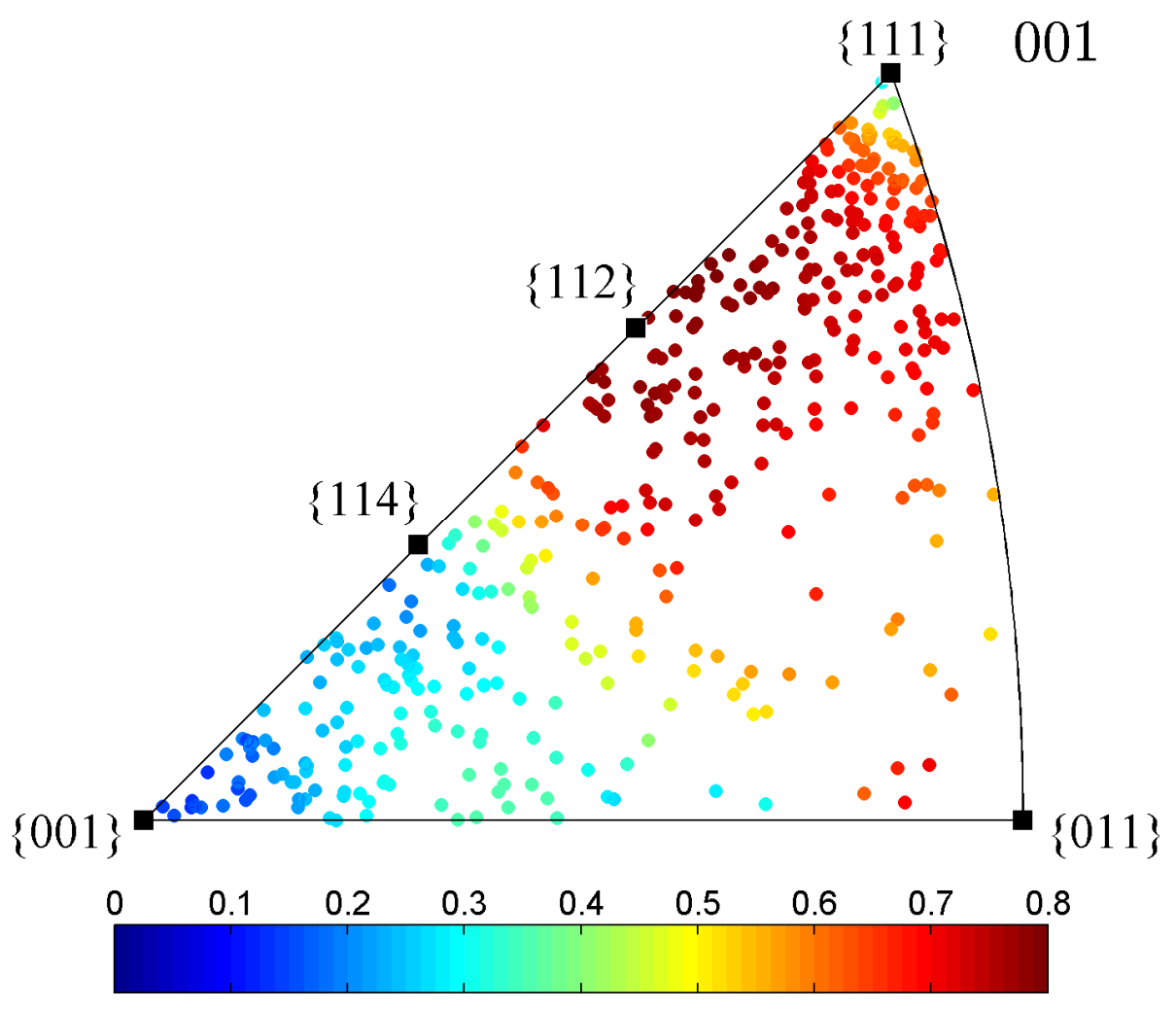

(a) rotation rate SDs - FCC

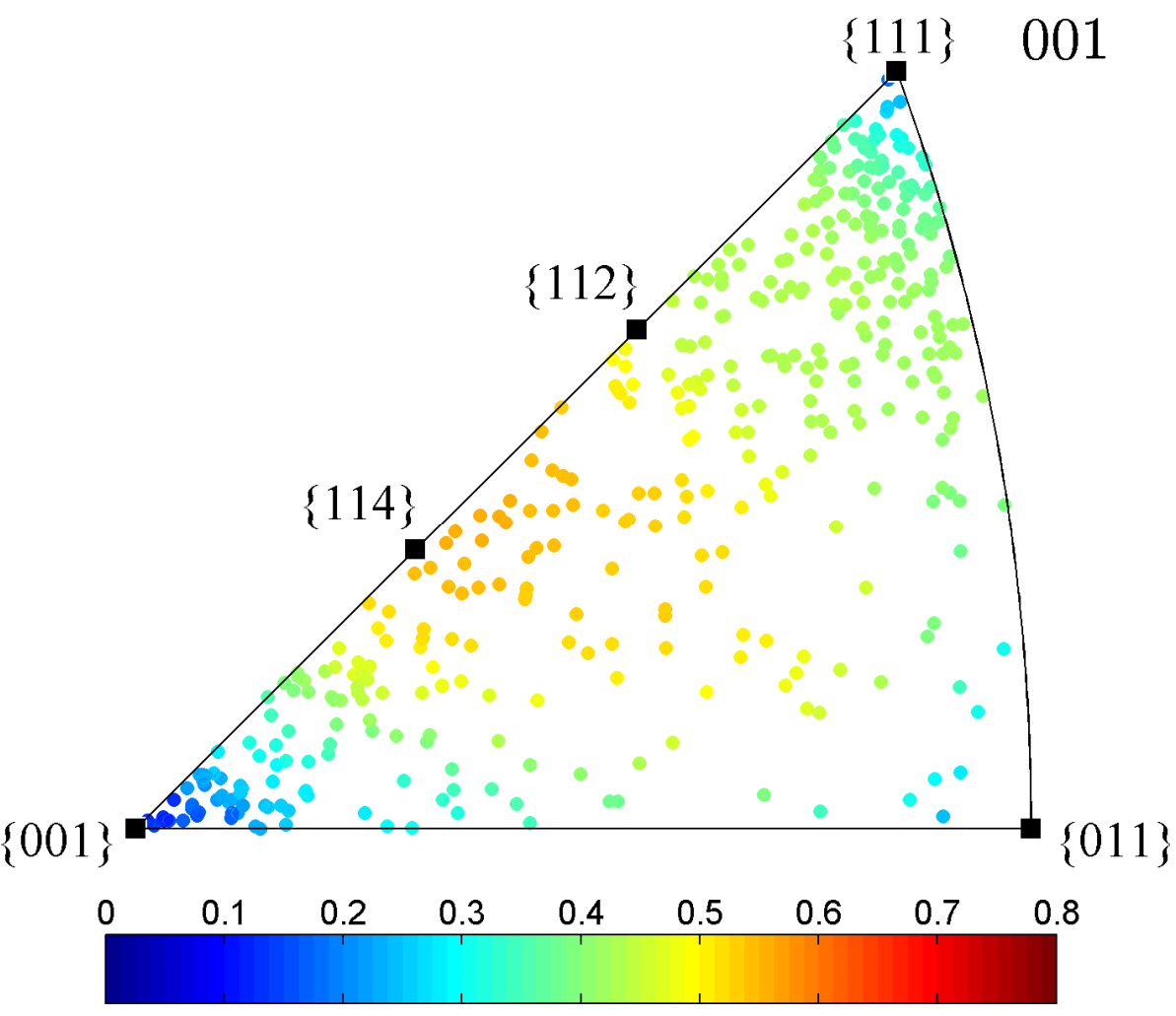

(b) rotation rate SDs - BCC

Figure 4: VPSC predictions of the per-grain geometric mean SD of lattice rotation rate distributions, $\mathrm{SD}^{(\mathrm{r})}(\dot{\omega}) / \overline{\dot{\omega}}$ (see Eq. (26)), after $25 \%$ tensile strain, considering texture and grain morphology evolution (average fluctuations of lattice rotation rates calculated using the full expression for non-spherical grains, Eq. (23)). Cases of: a) fcc polycrystal deforming in tension by $\{111\}<110>$ octahedral slip, and b) bcc polycrystal with spherical grains, deforming in compression by $\{110\},\{112\},\{123\}<111>$ pencil glide. 


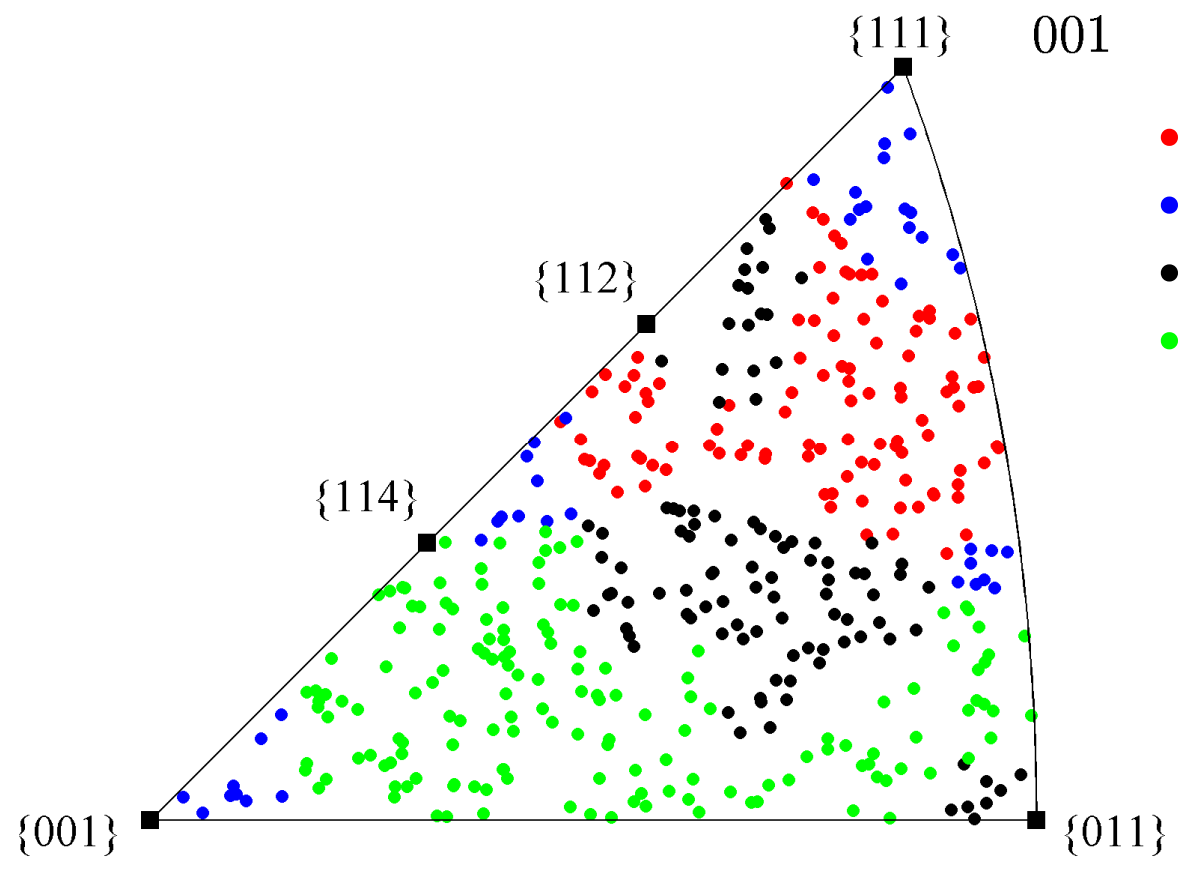

(a) initial

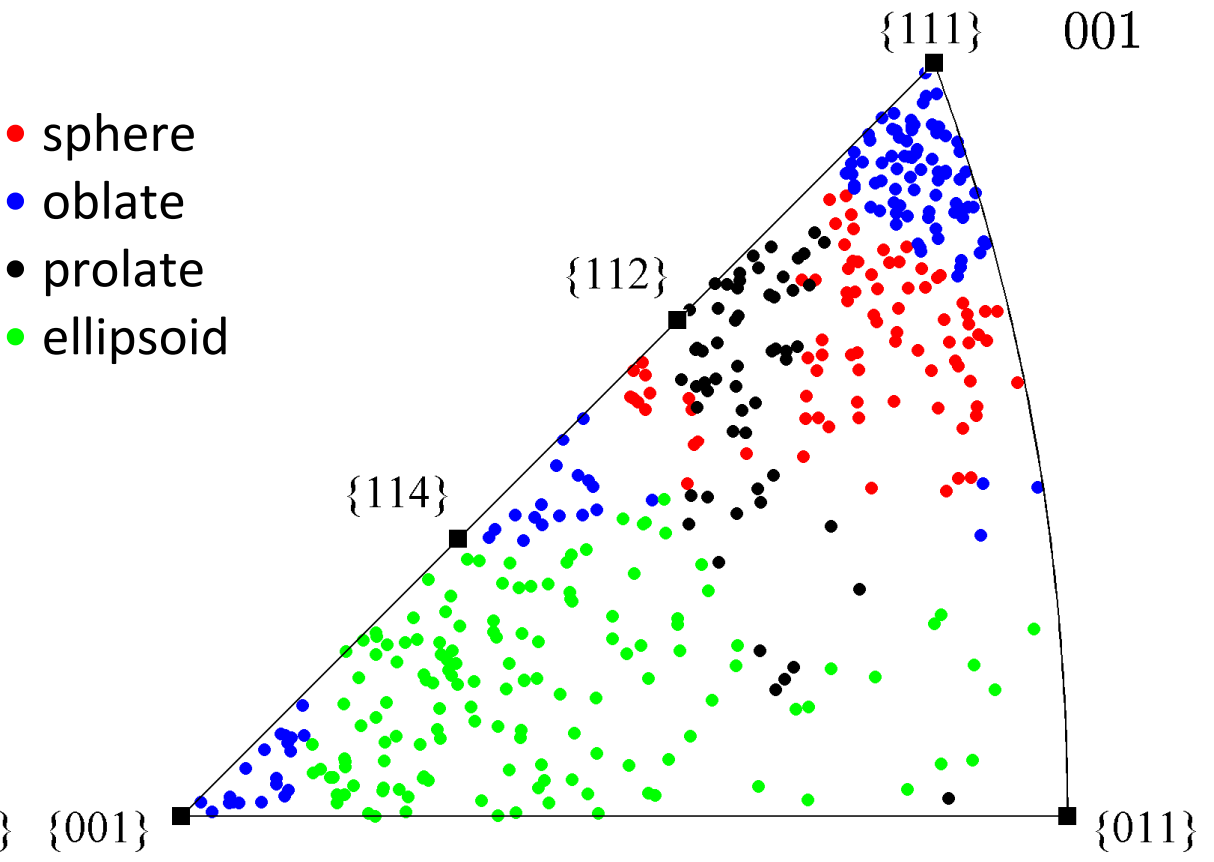

(b) after $25 \%$ strain

Figure 5: VPSC predictions of the shape of the distributions of lattice rotation rates, according to the ratios between the three $\mathrm{SD}_{\dot{i}}^{(\mathrm{r})}(\dot{\omega})$ (see Table 3), for an fcc polycrystal deforming in tension. Cases of: (a) initial texture, with random orientations and spherical grains, (b) after $25 \%$ strain accounting for texture and grain morphology evolution. 


\section{Tables}

\begin{tabular}{|l|l|l|l|l|l|l|}
\hline \multirow{2}{*}{} & \multicolumn{2}{|l|}{ VPSC } & \multicolumn{2}{l|}{ VPFFT } \\
\cline { 2 - 7 } & Minimum & Mean & Maximum & Minimum & Mean & Maximum \\
\hline$\langle\dot{\omega}\rangle^{(\mathrm{r})} / \overline{\dot{\omega}}$ & 0.140 & 1.000 & 1.768 & 0.105 & 1.000 & 1.851 \\
\hline $\mathrm{SD}^{(\mathrm{r})}(\dot{\omega}) / \overline{\dot{\omega}}$ & 0.117 & 0.553 & 0.775 & 0.386 & 0.557 & 0.680 \\
\hline
\end{tabular}

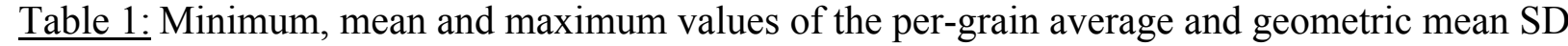
(Eq. (26)) predicted by VPSC and VPFFT

\begin{tabular}{|l|l|l|l|l|l|l|}
\hline \multirow{2}{*}{} & \multicolumn{2}{|l|}{ VPSC } & \multicolumn{2}{l|}{ VPFFT } \\
\cline { 2 - 7 } & Minimum & Average & Maximum & Minimum & Average & Maximum \\
\hline $\mathrm{SD}_{1}^{(\mathrm{r})}(\dot{\omega}) / \overline{\dot{\omega}}$ & 0.555 & 0.993 & 1.397 & 0.540 & 0.874 & 1.181 \\
\hline $\mathrm{SD}_{2}^{(\mathrm{r})}(\dot{\omega}) / \overline{\dot{\omega}}$ & 0.345 & 0.573 & 0.932 & 0.315 & 0.545 & 0.855 \\
\hline $\mathrm{SD}_{3}^{(\mathrm{r})}(\dot{\omega}) / \overline{\dot{\omega}}$ & 0.005 & 0.357 & 0.633 & 0.180 & 0.381 & 0.633 \\
\hline
\end{tabular}

Table 2: Minimum, mean and maximum values of the three individual SDs (Eq. (25)) of the pergrain rotation rate distributions, predicted by VPSC and VPFFT.

\begin{tabular}{|c|c|c|}
\hline & $\mathrm{SD}_{1}^{(\mathrm{r})} / \mathrm{SD}_{2}^{(\mathrm{r})}<1.5$ & $\mathrm{SD}_{1}^{(\mathrm{r})} / \mathrm{SD}_{2}^{(\mathrm{r})}>1.5$ \\
\hline $\mathrm{SD}_{2}^{(\mathrm{r})} / \mathrm{SD}_{3}^{(\mathrm{r})}<1.5$ & "sphere" & "prolate" \\
\hline $\mathrm{SD}_{2}^{(\mathrm{r})} / \mathrm{SD}_{3}^{(\mathrm{r})}>1.5$ & "oblate" & "ellipsoid" \\
\hline
\end{tabular}

Table 3: Classification of the shape of distributions, as a function of the relative values of the 3 standard deviations, according to Krog-Pedersen et al. [33]. 


\section{Graphical Abstract}

\section{VPSC predictions of average intragranular rotation rate fluctuations}

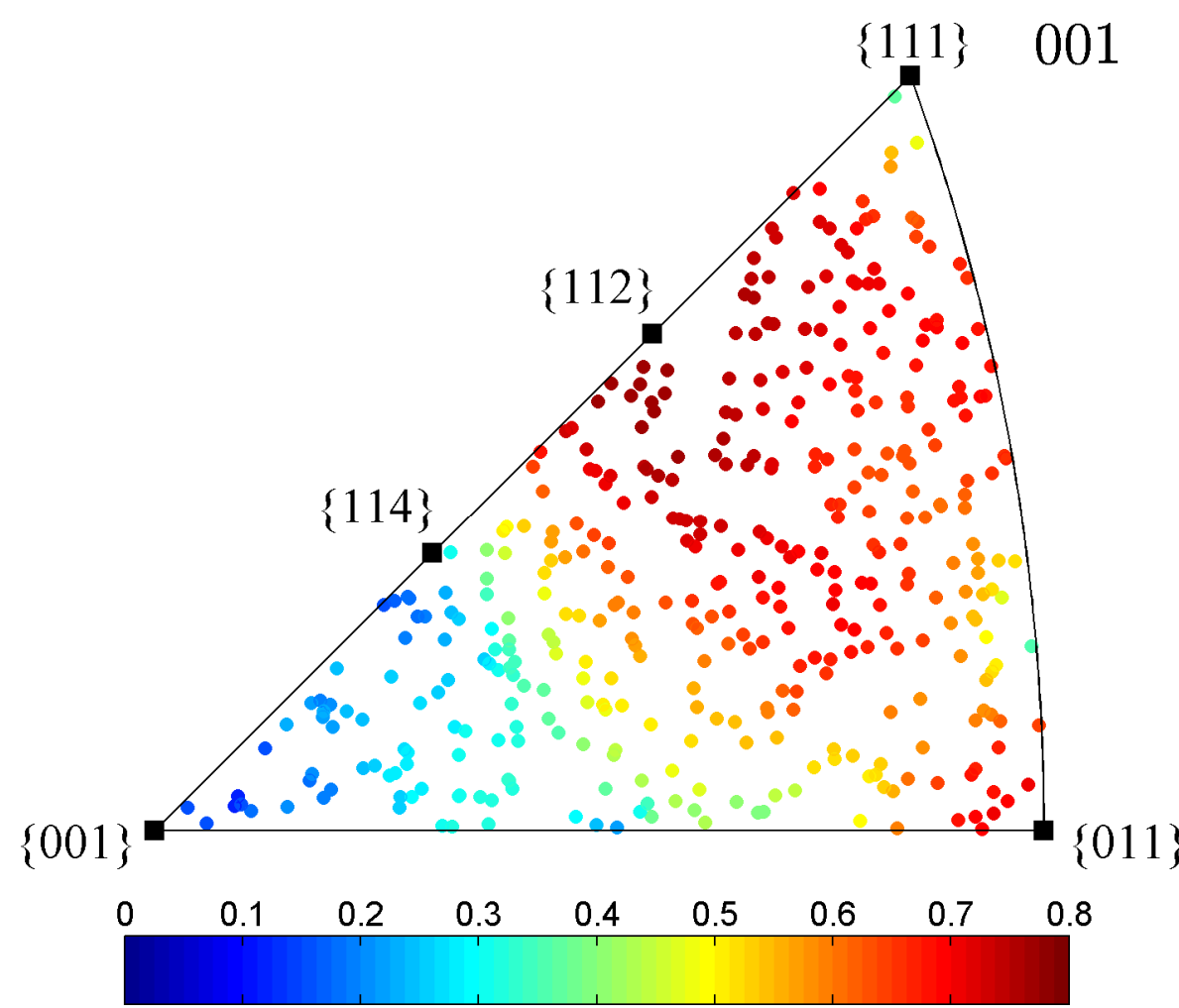

(a) rotation rate standard deviations for FCC in tension: $\max$ at $<112>$

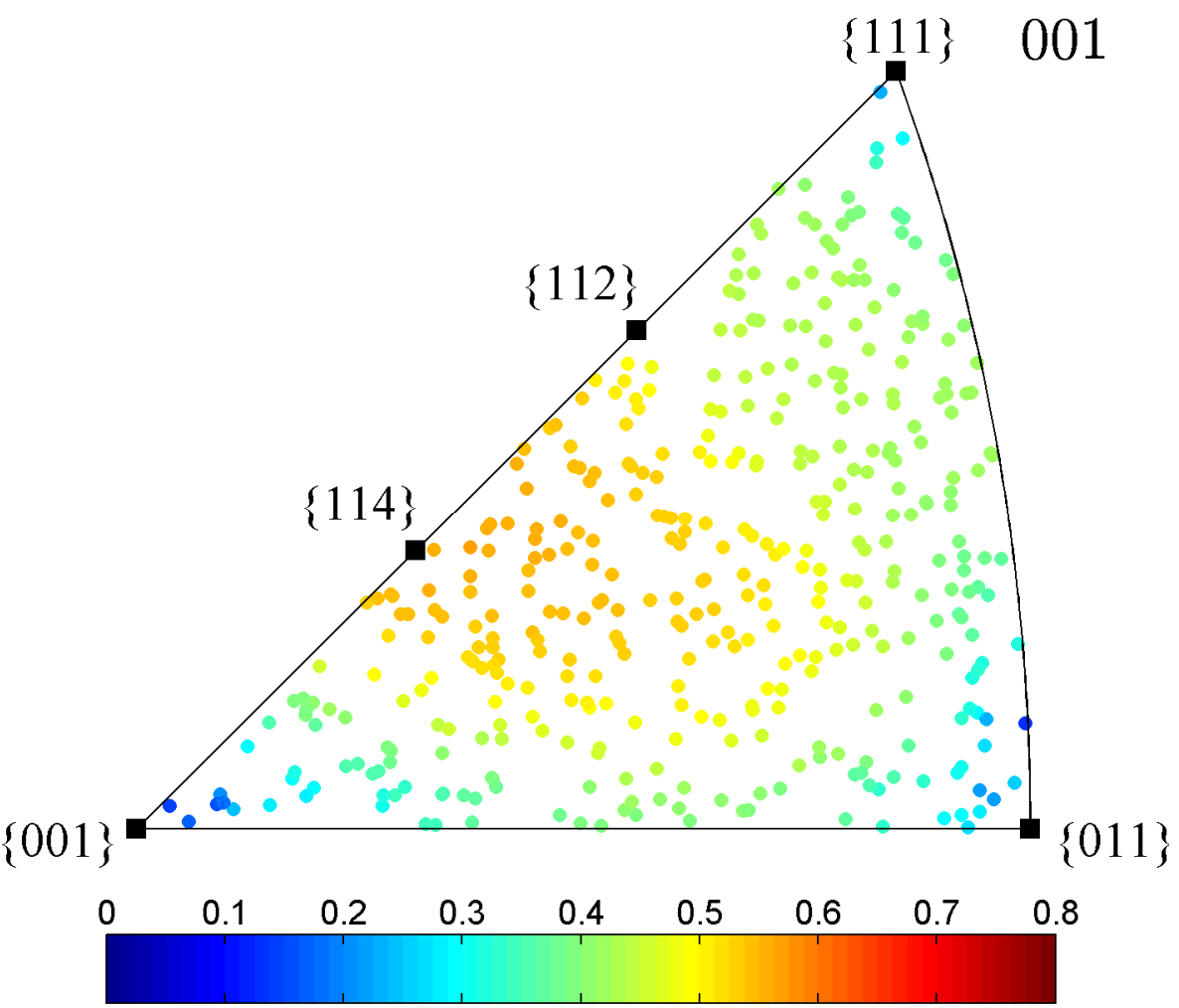

(b) rotation rate standard deviations for BCC in compression: max at <114> 